\title{
Effects of Moisture Deficit on the Yield of Cowpea Genotypes in the Guinea Savannah of Northern Ghana
}

\author{
Damba Yahaya ${ }^{1,2^{*}}$, Nicholas Denwar ${ }^{3}$, Matthew W. Blair ${ }^{1}$ \\ ${ }^{1}$ Department of Agricultural and Environmental Sciences, Tennessee State University, Nashville, TN, USA \\ ${ }^{2}$ Department of Biotechnology, University for Development Studies, Tamale, Ghana \\ ${ }^{3}$ Council for Scientific and Industrial Research, Savannah Agricultural Research Institute, Tamale, Ghana \\ Email: *yahayadamba@gmail.com, mwbeans@gmail.com, mblair@tnstate.edu
}

How to cite this paper: Yahaya, D., Denwar, N. and Blair, M.W. (2019) Effects of Moisture Deficit on the Yield of Cowpea Genotypes in the Guinea Savannah of Northern Ghana. Agricultural Sciences, 10, 577-595.

https://doi.org/10.4236/as.2019.104046

Received: March 12, 2019

Accepted: April 23, 2019

Published: April 26, 2019

Copyright $\odot 2019$ by author(s) and Scientific Research Publishing Inc. This work is licensed under the Creative Commons Attribution International License (CC BY 4.0).

http://creativecommons.org/licenses/by/4.0/

\begin{abstract}
Cowpea is multipurpose, leguminous, high protein crop in the tropics that provides food for humans and fodder for animals. The crop adds nitrogen and other nutrients to the soil through symbiotic relationship with rhizobia and direct decomposition of cowpea by-products. Despite its multiple benefits for humankind, the yield of cowpea is far below its potential and its production in the crop's birthplace of Africa is especially affected by abiotic factors. Soil moisture deficit is one of the main abiotic factors that affect the yield of cowpea in the semi-arid tropics, including the Sahelian and Guinea Savannah regions in West Africa. Even though cowpea is a drought tolerant legume, different genotypes respond differently to drought, resulting in up to $100 \%$ or more yield increases in the case of resistant genotypes or $50 \%$ or more yield loss in case of susceptible types. Mitigating the effect of soil moisture deficit on cowpea production requires selection of genotypes that can withstand drought. With this in mind, the goal of this study was to identify drought tolerant cowpea germplasm for the Savannah region of Northern Ghana using cultivated genotypes from the United States Department of Agriculture (USDA) tested with and without irrigation at the Bontanga irrigation facility during the dry season in 2018. Fifty genotypes were used, which included 45 imported from USDA and five (5) local genotypes from the Savannah Agriculture Research Institute (SARI). The experiment had $2 \times$ 50 factorial treatments (irrigation $\times$ genotypes) and consisted of randomized complete block design with three (3) replications per treatment. Two (2) watering regimes were introduced namely, drought stressed (no irrigation) and non-stressed/control (irrigated). Morpho-physiological, phenological and yield data were taken on the cowpeas evaluated with drought tolerance as-
\end{abstract}


sessed based on grain yield data and derived indices. All parameters measured showed significant differences $(p \leq 0.05)$ except for the number of branches per plant. Genotypes PI339600, PI527263, PI527302, PI582793, PI582867 and SARI-6-2-6 produced high grain yields under both drought stress and non-stress conditions. These genotypes could be exploited for future breeding programs for developing drought tolerant cowpea varieties for the savannah ecology and other areas with similar environmental conditions.

\section{Keywords}

Tolerance, Cowpea, Stress, Selection, Drought

\section{Introduction}

The cowpea [Vigna unguiculata (L.) Walp.] is a vital crop for Africa. It is predominantly grown by small-scale farmers in West Africa, where the crop is crucial to the livelihood and health of the economies and societies of most Sahelian countries from Nigeria and Chad in the east to Senegal in the west [1]. The grain and leaves of cowpea are highly nutritive, serving as a cheap source of protein for rural and urban dwelling consumers [2]. The cowpea grain contains about $25 \%$ protein and $64 \%$ carbohydrate and substantial quantities of fiber, minerals and vitamins [3]. The grains, leaves or fodder and food processed by-products from the harvest are sold, proving economic benefits for farming households in the region. The crop is often called a hunger crop since it matures early, which allows the edible leaves to be used during the hunger-prone part of the season when food reserves from previous harvest have been depleted and recently planted seeds of other farm crops are not yet ready for harvest. Aside from the grain which is an important human food [4] [5] [6], farmers also sell the fodder of cowpea for animal production. The rotation of cowpeas and livestock create a virtuous circle of legume and grazing animals, with cowpeas serving as green manure or cover crop to improve soil fertility and control erosion throughout the rainy season [5], goats and cattle are feeding on the stems during the dry season. The crop is also capable of fixing atmospheric nitrogen through its root nodules to enhance the nutrient status of the soil for the next crop in a rotation [6] [7]. Cowpea is adapted to the drier and hotter regions of the tropics and subtropics due to its drought tolerant qualities [8]. The crop is a healthy food legume that is considered as an important complement to soybean or groundnut that is high in oil content but lower in fiber [9].

In Ghana, cowpea ranks second to groundnut in terms of consumption [10] [11]. The cultivation of cowpea in Ghana is concentrated in five regions of the country namely Northern, Upper West, Upper East, Brong Ahafo and Ashanti regions [12]. The Northern region is the highest producer of cowpea and Yendi municipality is the highest producer of cowpea among the districts in the 
Northern region [12]. However, despite its widespread cultivation and importance, the productivity of cowpea in West Africa is very low. The normal typical yields across the region range from 100 to $500 \mathrm{~kg} / \mathrm{ha}$ [13]. According to [12], the average yields on farmers' fields in Ghana are between 400 and $600 \mathrm{~kg} / \mathrm{ha}$ as compared to values of 1600 to $2500 \mathrm{~kg} / \mathrm{ha}$ of potential yield recorded on research fields. The yield gap is attributed to biotic and abiotic factors affecting the crop's production in the tropics and sub tropics of the world.

Globally, increasing human population has led to higher demands for increased agricultural productivity [14]. The challenges to achieving higher crop yields is further compounded by drought and heat stress resulting from climate change [15]. Terminal and intermittent drought are known to cause substantial crop yield reduction due to their negative impacts on plant growth, physiology and reproduction [16]. The main effect of drought stress is the reduction of crop yield [17] [18] [19] through reduction in biomass [20] and seed weight. Shoot and root growth by plants are also limited by terminal and intermittent drought [21]. Drought is a complex phenomenon [22] dependent on the type of soil and rainfall patterns on agricultural lands but mostly is always responsible for major yield losses in the case of cowpea. The effect of drought varies with the duration and intensity of moisture deficits resulting from irregular or shortage of normal rainfall or late rains and late plantings that are affected by the prolonged drought of the dry season [23].

Cowpea is known to be a drought tolerant crop [24]. However, the crop still suffers from yield losses from intermittent and terminal drought in its main growing environments. As a result, genotypes that can grow and produce appreciable yield under drought conditions are required for increased yields. Researchers and plant breeders have made efforts to identify cowpea varieties with enhanced levels of drought and heat tolerance, as well as high biological nitrogen fixation [6] but generally this work has been conducted with local germplasm rather than introduced genotypes. The aims of this study were: 1) to introduce cowpea accessions into Northern Ghana from the USDA germplasm collection, as this is publicly available set of plant introductions from around the world; and 2) to identify drought tolerant cultivars among the USDA genotypes compared to local control varieties for use in future breeding programs in order to gain maximum genetic recombination and development of drought tolerant varieties suitable for Ghana and other African countries. Given the importance of cowpea in ensuring food and nutritional security in many developing countries, there is the need to develop and/or select available germplasm for improved yields.

\section{Materials and Methods}

\subsection{Experimental Site}

The research was conducted at the Bontaga station field in the Kumbungu district within the guinea savannah ecology in the northern region of Ghana. Bon- 
taga is located at western part of Kumbungu on latitude 9.583884 and longitude -1.023843 , and at an altitude of $116 \mathrm{~m}$ above sea level. Planting of the cowpea genotypes was done on $3^{\text {rd }}$ February 2018 during the dry season. The Bontaga station has a water source and irrigation facilities, allowing different levels of irrigated treatments to be compared at the same site. The area has a unimodal rainfall of $1100 \mathrm{~mm}$ per annum which occurs between May and October each year [25]. The soil being a sandy loam does not retain water well.

\subsection{Source of Cowpea Seeds Used}

The experiment included a total of 50 cowpea genotypes. Of these, 45 genotypes were selected from United States Department of Agriculture (USDA) germplasm and five (5) from the Savannah Agricultural Research Institute (SARI), Ghana. The USDA genotypes were all plant introductions (PIs) selected based on their survival and yield potential under drought stress from an earlier seed multiplication field trial.

\subsection{Irrigation Levels Used and Experimental Design}

The experiment involved the 50 genotypes and two levels of water supply. A non-stress (NS) treatment was based on full supply of irrigation water (every 3 days) throughout the growing period. This treatment was considered as control for high productivity compared to a drought stress (DS) treatment which involves the terminal withdrawal of irrigation water at flowering. The combination of genotypes $x$ irrigation regimes resulted in 100 treatment combinations. The treatments were planted as a randomized complete block design with three (3) replications per treatment. Seeds were planted on $2 \mathrm{~m} \times 1.2 \mathrm{~m}$ plots using inter and intra row spacings of $60 \mathrm{~cm}$ and $20 \mathrm{~cm}$, respectively. Weeds were manually controlled.

\subsection{Soil Data}

Before planting, soil samples were collected from holes dug from the surface to a $30 \mathrm{~cm}$ depth across 5 different points chosen at random from the experimental site. The soil samples were mixed, and subsamples analyzed for chemical properties such as $\mathrm{pH}\left(\mathrm{H}_{2} \mathrm{O}\right)$, organic carbon [26], total nitrogen (Kjeldahl method), phosphorus (P) concentration using Bray-2 [27], K, Na, Ca, Mg, S concentrations were also estimated. Finally, cation exchange capacity (CEC) was measured using ammonium acetate method according to [28]. Soil texture was also determined.

\subsection{Morpho-Physiological Plant Data}

The morpho-physiological data on each cowpea genotypes were monitored by recording the following parameters: 1) plant height (PLHT), measured at mid pod filling stage on five plants per plot using a meter stick; 2) number of branches per plant (NBP); 3) number of leaves per plant (NLP); 4) number of 
pods per plant (PDPL) were measured by randomly selecting five plants per plot; 5) dry shoot weight (DSW) was measured on two (2) plants after oven-drying at $65^{\circ} \mathrm{C}$ for 48 hours. Means were calculated from two plants per plot for each measurement.

\subsection{Phenological Data}

Days to flowering was recorded when $50 \%$ of the plants in a plot had at least one opened flower. Days to maturity was calculated based on the number of days from sowing to physiological maturity of at least 90 percent of the plants in a plot.

\subsection{Yield and Yield Components}

In addition to pods per plant (PDPL), the following yield traits were measured total seed yield (YLDH) and hundred (100) seed weight (100 SW), both recorded on per plot basis using sensitive digital balance. The $100 \mathrm{SW}$ was based on a random sample taken from the total yield. Finally, yield was corrected based on seed moisture content determined with a seed moisture meter (Dickey John Corporation, USA). The grain yield per plot was converted to yield per hectare using plant population after adjusting to $12 \%$ moisture content.

\subsection{Drought Indices}

Eight (8) indices were derived from the original data by comparing NS and DS treatments.

1) Drought Intensity Index (DII) $=1-\frac{\mathrm{YDS}}{\mathrm{YNS}}$

2) Stress Susceptibility Index (SSI) $=\frac{1-\left(\frac{\mathrm{YDSi}}{\mathrm{YNSi}}\right)}{1-\left(\frac{\mathrm{YDS}}{\mathrm{YNS}}\right)}$ or $\frac{1-\left(\frac{\mathrm{YDS}}{\mathrm{YNS}}\right)}{\mathrm{DII}}$

(2) [29]

3) Stress Tolerance Index $(\mathrm{STI})=\frac{\mathrm{YDSi} * \mathrm{YNSi}}{(\mathrm{YNS})^{2}}$ or $\frac{\mathrm{YDSi} * \mathrm{YNSi}}{\mathrm{YNS} * \mathrm{YNS}}$

(3) $[30]$

4) Mean Productivity (MP) $=\frac{\text { YDSi }+ \text { YNSi }}{2}$

(4) $[31]$

5) Geometric Mean Productivity $($ GMP $)=\sqrt{\text { YDSi } \times \text { YNSi }}$

6) Yield Index (YI) $=\frac{Y d s i}{Y s}$

7) Yield Reduction Rate $(\mathrm{YRR})=\left(\frac{\mathrm{YNSi}-\mathrm{YDSi}}{\mathrm{YNSi}}\right) \times 100$

8) Yield Stability Index $(Y S I)=\frac{\text { YDSi }}{\text { YNSi }}$

where DS =drought stress, NS = non-stress, DSi = drought stressed individual, $\mathrm{NSi}=$ non stressed individual, $\mathrm{YDSi}=$ yield of drought stressed individual and YNSi $=$ yield of non-stressed individual. 


\subsection{Statistical Analysis}

Phenotypic data collected from the field experiment were subjected to analysis of variance using GENSTART version 12 after the data was tested for normality. Means of treatments that were significantly different from each other were separated using a Fisher's protected least significant difference (LSD) means comparison method.

\section{Results}

\subsection{Weather and Soil Conditions}

Relative humidity, rainfall and average temperature during the experimental period are presented in Figure 1. The soil was sandy loam in texture with a $\mathrm{pH}$ of 5.89 and low organic matter. Organic carbon was found to be $0.82 \%$, Nitrogen was $0.08 \%$, phosphorus was $7.15 \mathrm{mg} / \mathrm{kg}$, potassium was measured at 0.22 $\mathrm{cmol} / \mathrm{kg}$, and the cation exchange capacity of the soil was $5.17 \mathrm{cmol} / \mathrm{kg}$. Rainfall was scant during the entire experiment with one event of less than $10 \mathrm{~mm}$ at 70 days after planting. Average Daily temperatures ranged from $29^{\circ} \mathrm{C}$ to $32^{\circ} \mathrm{C}$ during the growing season with minimum and maximums of $26.25^{\circ} \mathrm{C}$ and $34.65^{\circ} \mathrm{C}$ respectively. Relative humidity was low (25\%) in February, increasing in March to $50 \%$ and April to $70 \%$. All experimental plots were supplied with water to field capacity until emergence of flower buds. Thereafter, moisture stress was imposed on the appropriate DS plots, while watering was continued for the NS plots.

\subsection{Genetic Variability of Cowpea Genotypes in Response to Water Stress}

ANOVAs showed that the genotypes showed significant variation in the number of days to flowering and maturity, dry shoot weight, number of branches and leaves, number of pods per plant, hundred seed weight and grain yield (Table 1).

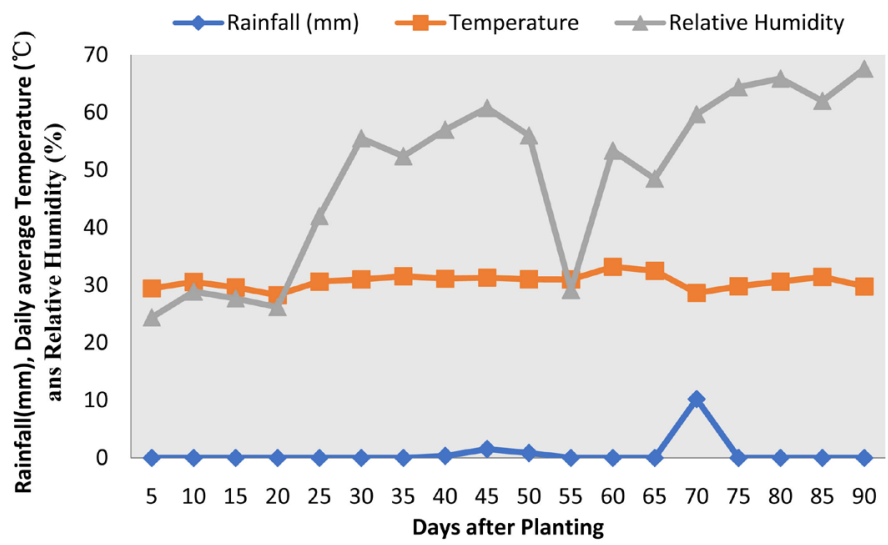

Figure 1. Climatological conditions of rainfall, relative humidity and average daily temperature across the growing season for 50 cowpea genotypes at the site of Bontaga field station in the Kumbungu district within the guinea savannah ecology in the northern region of Ghana. 
Table 1. Analyses of variance results for 50 cowpea genotypes evaluated at two irrigation levels, with means squares of biomass, yield and yield component traits and reduction of trait means under drought stress (DS) versus non-stress (NS) conditions.

\begin{tabular}{ccccccccc}
\hline Source of variation & DF & DM & DSW & HSW & NBplt & NLplt & NPplt & YLDha \\
\hline Genotype $(\mathrm{df}=49)$ & $24.28^{* * *}$ & $42.47^{* * *}$ & $3031.3^{* * *}$ & $78.76^{* * *}$ & $2.46^{* * *}$ & $2166^{* * *}$ & $168.30^{* * *}$ & $11,658,008^{* * *}$ \\
Treatment $(\mathrm{df}=1)$ & $1430.08^{* * *}$ & $12,168.10^{* * *}$ & $52,323.9^{* * *}$ & $12.41^{* * *}$ & $0.19 \mathrm{~ns}$ & $70334.1^{* * *}$ & $12,802.07^{* * *}$ & $100,312,405^{* * *}$ \\
$\mathrm{G}^{*} \mathrm{~T}(\mathrm{df}=49)$ & $24.15^{* * *}$ & $36.79^{* * *}$ & $1075.5^{* * *}$ & $7.94^{* * *}$ & $1.86^{* * *}$ & $673.3^{* * *}$ & $84.68^{* * *}$ & $1,244,491^{* * *}$ \\
Residual $(\mathrm{df}=198)$ & 2.79 & 2.68 & 165.9 & 1.24 & 0.49 & 217.8 & 9.61 & 663,390 \\
Mean & 43.34 & 64.52 & 65.05 & 11.23 & 3.92 & 67.56 & 17.43 & 2090 \\
SED & 1.67 & 1.64 & 12.88 & 1.11 & 0.70 & 14.76 & 3.1 & 814.5 \\
CV & 3.9 & 2.5 & 19.8 & 9.9 & 17.9 & 21.8 & 17.8 & 39.0 \\
NS & 45.52 & 70.89 & 78.25 & 11.44 & 3.94 & 82.88 & 23.96 & 2668.12 \\
DS & 41.15 & 58.15 & 51.84 & 11.03 & 3.89 & 52.25 & 10.90 & 1511.7 \\
\% Reduction & 9.60 & 17.97 & 33.75 & 3.58 & 1.27 & 36.96 & 54.51 & 43.33 \\
\hline
\end{tabular}

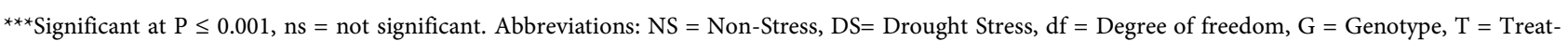
ment, DF = Days to flowering, DM = Days to maturity, DSW $=$ Dry shoot weight, HSW $=$ Hundred seed weight, NBplt $=$ Number of branches per plant, NLplt $=$ Number of leaves per plant, NPplt $=$ Number of pods per plant and YLDha $=$ Grain yield per hectare

All measured traits were significantly affected by the drought treatment except for the number of branches per plant. The interaction between watering regime and genotypes showed marked influences on all the traits measured. The interaction between water regime and genotypes was highly significant, with drought stress consistently reducing the performance of the genotypes tested.

\subsection{Physiological Response of Cowpea Genotypes to Water Stress}

The number of days to flowering and maturity of the genotypes was influenced by watering regime. The drought stressed genotypes consistently flowered and matured earlier (Table 2). Flowering among genotypes ranged from 38 to 52 days after sowing, with a mean of 43 days. The number of days to maturity ranged from 56 to 82 days after sowing, with a mean of 65 days. Genotypes PI583182 and PI583209 flowered earlier while PI582867 flowered late. As a result, genotypes PI583182 and PI293463 matured earlier compared to other genotypes while genotypes SONGOTRA and PI354466 matured late. Exposing the test genotypes to drought caused $9.6 \%$ and $18.0 \%$ reduction in the number of days to flowering and maturity, respectively.

The growth and development of the plants varied significantly among genotypes and was also markedly influenced by the watering regime used. For example, the number of leaves showed significant variation between treatments, with values ranging from 22 to 125 leaves per plant. The DS plants recorded 52.3 lower number of leaves per plant compared to control plants under NS conditions. However, there was no significant effect of watering regime on the number of branches per plant and only $1.3 \%$ reduction was observed between treatments despite significant genotypic differences for this trait. 
Table 2. The means for days to flowering (DF), days to maturity (DM), number of branches per plant (NBplt) and number of leaves per plant (NLplt) for 50 genotypes of cowpeas grown in Northern Ghana under drought stress (DS) and non-stress (NS) conditions. Values (Mean \pm SE) followed letters in a column are significantly different at $\mathrm{p} \leq 0.05$.

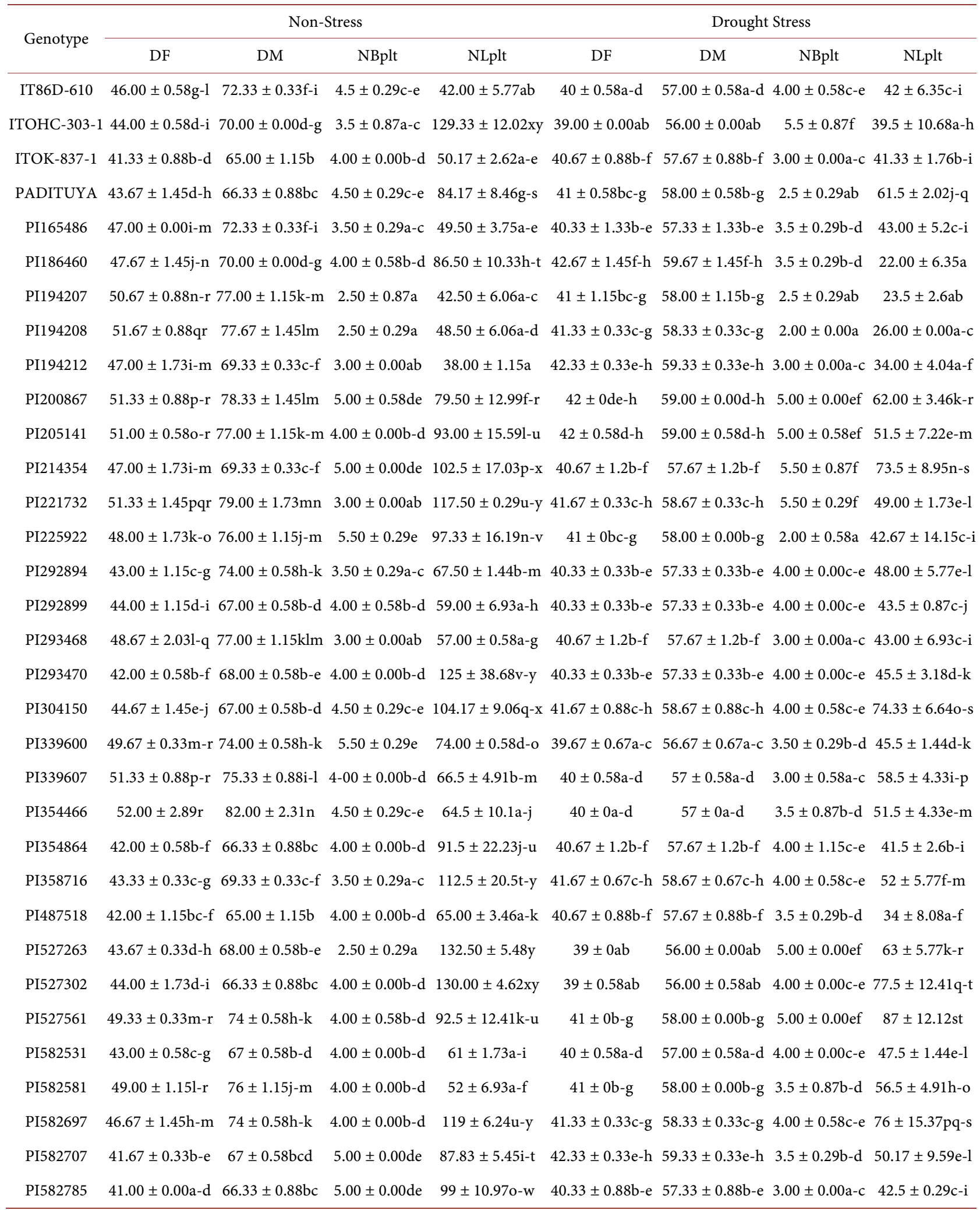




\begin{tabular}{|c|c|c|c|c|c|c|c|c|}
\hline PI582789 & $43.00 \pm 1.15 c-g$ & $66.33 \pm 0.88 b c$ & $5.00 \pm 0.58 \mathrm{de}$ & $78.33 \pm 5.04 f-r$ & $41.33 \pm 0.88 c-g$ & $58.33 \pm 0.88 \mathrm{c}-\mathrm{g}$ & $4.00 \pm 0.58 c-e$ & $46.17 \pm 7.58 \mathrm{~d}-\mathrm{k}$ \\
\hline PI582793 & $45.00 \pm 1.73 \mathrm{f}-\mathrm{k}$ & $67 \pm 0.58 \mathrm{bcd}$ & $3.5 \pm 0.87 a-c$ & $75.17 \pm 4.8 \mathrm{~d}-\mathrm{p}$ & $43.67 \pm 0.33 h$ & $60.67 \pm 0.33 h$ & $3.00 \pm 0.58 \mathrm{a}-\mathrm{c}$ & $47.33 \pm 5.78 \mathrm{e}-1$ \\
\hline PI582853 & $46.00 \pm 1.73 \mathrm{~g}-1$ & $74 \pm 0.58 \mathrm{~h}-\mathrm{k}$ & $3.00 \pm 0.00 \mathrm{ab}$ & $54 \pm 0.58 \mathrm{a}-\mathrm{f}$ & $40.67 \pm 1.2 b-f$ & $57.67 \pm 1.2 b-f$ & $4.5 \pm 0.29 \mathrm{~d}-\mathrm{f}$ & $29 \pm 4.62 \mathrm{a}-\mathrm{d}$ \\
\hline PI582867 & $51.67 \pm 0.33 \mathrm{qr}$ & $77 \pm 1.15 \mathrm{k}-\mathrm{m}$ & $4.00 \pm 0.00 \mathrm{~b}-\mathrm{d}$ & $126 \pm 17.32 \mathrm{w}-\mathrm{y}$ & $43.67 \pm 0.88 h$ & $60.67 \pm 0.88 h$ & $4.5 \pm 0.29 \mathrm{~d}-\mathrm{f}$ & $95 \pm 9.81 \mathrm{t}$ \\
\hline PI583205 & $42.00 \pm 0.00 \mathrm{~b}-\mathrm{f}$ & $67 \pm 0.58 b-d$ & $4.00 \pm 0.00 \mathrm{~b}-\mathrm{d}$ & $106.17 \pm 11.12 \mathrm{r}-\mathrm{y}$ & $40.67 \pm 0.67 b-f$ & $57.67 \pm 0.67 b-f$ & $5.00 \pm 0.58 \mathrm{ef}$ & $79.83 \pm 1.83 \mathrm{r}-\mathrm{t}$ \\
\hline PI583209 & $42.67 \pm 0.88 c-f$ & $66.33 \pm 0.88 b c$ & $3.50 \pm 0.29 \mathrm{a}-\mathrm{c}$ & $98.5 \pm 7.090-w$ & $38 \pm 0.58 \mathrm{a}$ & $55.00 \pm 0.58 \mathrm{a}$ & $3.5 \pm 0.29 b-d$ & $57 \pm 13.28 \mathrm{~h}-\mathrm{o}$ \\
\hline PI583254 & $47.33 \pm 0.33 \mathrm{j}-\mathrm{m}$ & $73.00 \pm 0.58 g-j$ & $3.00 \pm 0.58 \mathrm{ab}$ & $102.5 \pm 0.29 p-x$ & $43 \pm 1.15 \mathrm{gh}$ & $60.00 \pm 1.15 \mathrm{gh}$ & $3.00 \pm 0.00 \mathrm{a}-\mathrm{c}$ & $53.5 \pm 5.48 \mathrm{~g}-\mathrm{m}$ \\
\hline PI610520 & $48.33 \pm 2.031-p$ & $77.0 \pm 1.15 \mathrm{k}-\mathrm{m}$ & $5.00 \pm 0.00 \mathrm{de}$ & $86.17 \pm 3.56 \mathrm{~h}-\mathrm{t}$ & $40 \pm 0.58 \mathrm{a}-\mathrm{d}$ & $57 \pm 0.58 \mathrm{a}-\mathrm{d}$ & $4.5 \pm 0.29 \mathrm{~d}-\mathrm{f}$ & $72.5 \pm 2.57 \mathrm{n}-\mathrm{s}$ \\
\hline PI339598 & $46.67 \pm 0.88 \mathrm{~h}-\mathrm{m}$ & $73.00 \pm 0.58 g-j$ & $4.50 \pm 0.29 c-e$ & $70 \pm 7.51 c-n$ & $41 \pm 1.15 b-g$ & $58 \pm 1.15 b c-g$ & $4.00 \pm 0.00 c-\mathrm{e}$ & $49.5 \pm 3.75 \mathrm{e}-1$ \\
\hline PI632777 & $45.00 \pm 1.15 \mathrm{f}-\mathrm{k}$ & $72.33 \pm 0.33 \mathrm{f}-\mathrm{i}$ & $4.50 \pm 0.29 c-e$ & $93.5 \pm 8.95 \mathrm{~m}-\mathrm{u}$ & $41.33 \pm 1.2 \mathrm{c}-\mathrm{g}$ & $58.33 \pm 1.2 \mathrm{c}-\mathrm{g}$ & $5.50 \pm 0.29 f$ & $55.5 \pm 11.84 \mathrm{~h}-\mathrm{n}$ \\
\hline PI632796 & $45.00 \pm 0.58 \mathrm{f}-\mathrm{k}$ & $71.00 \pm 0.00 \mathrm{e}-\mathrm{h}$ & $4.00 \pm 0.58 b-d$ & $76.67 \pm 9.21 \mathrm{e}-\mathrm{q}$ & $41 \pm 0.58 b-g$ & $58 \pm 0.58 b-g$ & $4.5 \pm 0.29 \mathrm{~d}-\mathrm{f}$ & $49.83 \pm 4.21 \mathrm{e}-1$ \\
\hline PI663009 & $41.33 \pm 0.33 b-d$ & $66.33 \pm 0.88 b c$ & $2.50 \pm 0.29 a$ & $65.5 \pm 7.52 \mathrm{a}-1$ & $41 \pm 0 b-g$ & $58.00 \pm 0.00 \mathrm{~b}-\mathrm{g}$ & $2.5 \pm 0.29 \mathrm{ab}$ & $36.5 \pm 2.6 a-g$ \\
\hline SARI-6-2-6 & $40.33 \pm 0.88 \mathrm{a}-\mathrm{c}$ & $68 \pm 0.58 b-e$ & $4 \pm 0.58 b-d$ & $84.33 \pm 6.77 \mathrm{~g}-\mathrm{s}$ & $43.67 \pm 0.33 h$ & $60.67 \pm 0.33 h$ & $5.00 \pm 0.00 \mathrm{ef}$ & $65.5 \pm 2.021 \mathrm{~lm}-\mathrm{r}$ \\
\hline SONGOTRA & $43.00 \pm 0.58 c-g$ & $74 \pm 0.58 \mathrm{hi}-\mathrm{k}$ & $3.5 \pm 0.29 \mathrm{a}-\mathrm{c}$ & $45.17 \pm 0.44 a-c$ & $48.67 \pm 0.33 \mathrm{i}$ & $65.67 \pm 0.33 i$ & $3.00 \pm 0.58 \mathrm{a}-\mathrm{c}$ & $33.33 \pm 6.36 \mathrm{a}-\mathrm{e}$ \\
\hline WANGKAE & $39.33 \pm 0.33 \mathrm{ab}$ & $66.33 \pm 0.88 b c$ & $3.5 \pm 0.29 a-c$ & $109 \pm 6.35 \mathrm{~s}-\mathrm{y}$ & $42.67 \pm 0.88 \mathrm{f}-\mathrm{h}$ & $59.67 \pm 0.88 f-h$ & $4.00 \pm 0.58 \mathrm{cde}$ & $79 \pm 2.31 \mathrm{q}-\mathrm{t}$ \\
\hline
\end{tabular}

$\mathrm{DF}=$ Days to flowering, $\mathrm{DM}=$ Days to maturity, NBplt $=$ Number of branches per plant, NLplt $=$ Number of leaves per plant.

\subsection{Biomass and Yield Responses of Cowpea Genotypes}

Large effects were observed of watering regime on biomass accumulation and yield traits. The biomass reduction was reflected in $33.8 \%$ lower dry shoot weight of the drought treated plants when compared to the control/non-stressed plants. Similarly, the number of pods per plant was negatively affected by watering regime, causing $54.5 \%$ pods reduction in DS plants compared to the NS plants (Table 1). Seed size as reflected by the 100SW trait showed less of a reduction of $3.9 \%$ even though the differences between DS and NS were highly significant. NS plants produced average seed weights of $11.44 \mathrm{~g} / 100$ seed while DS plants were $11.03 \mathrm{~g} / 100$ seed although this varied greatly between genotypes. The variety "Padituya" had notably large seed of 25.3 and $22.1 \mathrm{~g} / 100$ seed in the two conditions, while some genotypes had seed as small as 5 to $7 \mathrm{~g} / 100$ seed, especially under the DS condition (Table 3).

Overall yield per hectare was reduced on average by $43.3 \%$ comparing the NS and DS conditions. The average yield under well-watered condition was 2668.12 $\mathrm{kg} / \mathrm{ha}$ for the fifty genotypes but was only $1511.7 \mathrm{~kg} / \mathrm{ha}$ under DS. The calculated drought intensity index was 0.57 , with the stress negatively affecting the yield of the cowpeas tested. Drought tolerance and susceptibility indices were estimated for individual genotypes. Based on mean and geometric productivity, PI527561, PI582707, PI527302, PI304150, PI583205, PI632777, PI610520, PI339600, PI582867, PI205141, PI200867, PI582697, SARI-6-2-6, PI292899, PI582789 and PI527263 produced high yields, with values ranging from $2551.5 \mathrm{~kg} / \mathrm{ha}$ to 6158 $\mathrm{kg} / \mathrm{ha}$ (Table 4). 
Table 3. The means of dry shoot weight (DSW), number of pods per plant (NPplt), hundred seed weight (100SW) and yield in $\mathrm{kg} / \mathrm{ha}$ (YLDha) for 50 genotypes of cowpeas grown in Northern Ghana under drought stress (DS) and non-stress (NS) conditions. Values (Mean $\pm \mathrm{SE}$ ) followed letters in a column are significantly different at $\mathrm{p} \leq 0.05$.

\begin{tabular}{|c|c|c|c|c|c|c|c|c|}
\hline \multirow{2}{*}{ Genotype } & \multicolumn{4}{|c|}{ Non-Stress } & \multicolumn{4}{|c|}{ Stress } \\
\hline & DSW (g) & NPlt & HSW (g) & YLDha (Kg) & DSW (g) & NPlt & HSW (g) & YLDha (kg) \\
\hline ITOHC-303-1 & $63.49 \pm 0.60 \mathrm{e}-\mathrm{i}$ & $21.17 \pm 1.42 \mathrm{~b}-\mathrm{g}$ & $20.81 \pm 1.95 u$ & $3370.83 \pm 561.95 j-p$ & $73.74 \pm 6.67 \mathrm{~s}-\mathrm{w}$ & $17.92 \pm 0.510-\mathrm{s}$ & $23.00 \pm 1.15 y$ & $362.5 \pm 7.22 \mathrm{a}-\mathrm{e}$ \\
\hline ITOK-837-1 & $87.89 \pm 0.13 \mathrm{j}-\mathrm{q}$ & $27.00 \pm 0.00 \mathrm{~h}-1$ & $16.65 \pm 0.33 \mathrm{rs}$ & $753.75 \pm 4.09 \mathrm{ab}$ & $39.51 \pm 1.12 \mathrm{~d}-\mathrm{k}$ & $2.5 \pm 0.29 \mathrm{a}-\mathrm{c}$ & $17.08 \pm 1.64 \mathrm{x}$ & $349.17 \pm 0.96 a-e$ \\
\hline PADITUYA & $108.02 \pm 2.95 \mathrm{q}-\mathrm{t}$ & $33.00 \pm 1.73 \mathrm{mn}$ & $25.3 \pm 0.12 \mathrm{v}$ & $2154.17 \pm 28.56 \mathrm{c}-\mathrm{k}$ & $46.7 \pm 4.69 \mathrm{~g}-\mathrm{o}$ & $1.5 \pm 0.29 \mathrm{ab}$ & $22.14 \pm 0.36 y$ & $1695 \pm 217.881-n$ \\
\hline PI165486 & $25.31 \pm 1.28 \mathrm{a}$ & $18.00 \pm 0.87 a-e$ & $13.11 \pm 0.31 \mathrm{op}$ & $677.08 \pm 18.52 \mathrm{ab}$ & $24.93 \pm 4.54 a-f$ & $6 \pm 0.58 c-g$ & $12.4 \pm 0.28 \mathrm{r}-\mathrm{t}$ & $430.83 \pm 0.96 a-f$ \\
\hline PI186460 & $30.58 \pm 0.25 \mathrm{ab}$ & $13.75 \pm 0.66 a$ & $8.23 \pm 0.02 \mathrm{a}-\mathrm{f}$ & $512.92 \pm 3.13 a$ & $25.10 \pm 0.20 \mathrm{a}-\mathrm{f}$ & $1.33 \pm 0.33 \mathrm{ab}$ & $8.75 \pm 0.03 \mathrm{e}-\mathrm{k}$ & $145.83 \pm 1.44 a$ \\
\hline PI194207 & $20.59 \pm 1.48 \mathrm{a}$ & $17.25 \pm 1.15 \mathrm{a}-\mathrm{d}$ & $7.69 \pm 1.18 \mathrm{a}-\mathrm{c}$ & $780.56 \pm 283.42 a-c$ & $17.30 \pm 1.70 \mathrm{ab}$ & $6.5 \pm 1.04 \mathrm{c}-\mathrm{g}$ & $7.30 \pm 0.00 \mathrm{~b}-\mathrm{e}$ & $620 \pm 268.47 \mathrm{~d}-\mathrm{g}$ \\
\hline PI194212 & $42.65 \pm 6.37 a-e$ & $17.58 \pm 1.02 \mathrm{a}-\mathrm{d}$ & $7.81 \pm 0.17 a-d$ & $772.36 \pm 246.19 \mathrm{a}-\mathrm{c}$ & $16.13 \pm 0.22 \mathrm{a}$ & $15.17 \pm 0.17 \mathrm{~m}-\mathrm{q}$ & $7.69 \pm 0.44 b-f$ & $499.17 \pm 6.25 a-f$ \\
\hline PI200867 & $76.15 \pm 5.48 \mathrm{~g}-\mathrm{o}$ & $22.50 \pm 0.87 \mathrm{~d}-\mathrm{h}$ & $9.78 \pm 0.54 \mathrm{~d}-\mathrm{k}$ & $3692.92 \pm 42.11-q$ & $40.68 \pm 1.02 \mathrm{e}-1$ & $8.5 \pm 1.44 \mathrm{e}-\mathrm{j}$ & $11.09 \pm 0.19 \mathrm{n}-\mathrm{r}$ & $2838.33 \pm 1281.24 \mathrm{rs}$ \\
\hline PI205141 & $90.04 \pm 1.09 \mathrm{j}-\mathrm{q}$ & $20.25 \pm 1.30 \mathrm{~b}-\mathrm{f}$ & $16.7 \pm 0.53 \mathrm{rs}$ & $4622.92 \pm 1150.61 \mathrm{p}-\mathrm{t}$ & $22.57 \pm 5.67 a-d$ & $5.5 \pm 0.87 \mathrm{~b}-\mathrm{f}$ & $13.12 \pm 0.98 \mathrm{tu}$ & $1932.5 \pm 198.7 \mathrm{~m}-\mathrm{o}$ \\
\hline PI214354 & $98.04 \pm 4.460-\mathrm{s}$ & $27.50 \pm 0.50 \mathrm{~h}-\mathrm{m}$ & $8.5 \pm 0.02 \mathrm{a}-\mathrm{g}$ & $3253.33 \pm 881.9 j-p$ & $51.90 \pm 5.00 \mathrm{~h}-\mathrm{q}$ & $15.5 \pm 3.18 \mathrm{n}-\mathrm{r}$ & $8.39 \pm 0.04 \mathrm{~d}-\mathrm{h}$ & $1577.5 \pm 146.26 \mathrm{k}-\mathrm{m}$ \\
\hline PI221732 & $81.69 \pm 0.86 h-p$ & $28.5 \pm 1.73 \mathrm{i}-\mathrm{m}$ & $11.02 \pm 0.04 \mathrm{i}-\mathrm{n}$ & $2343.47 \pm 142.42 \mathrm{e}-1$ & $42.04 \pm 1.16 \mathrm{f}-\mathrm{m}$ & $10.00 \pm 1.15 \mathrm{~g}-1$ & $11.43 \pm 0.97 \mathrm{o}-\mathrm{s}$ & $1891.25 \pm 821.04 \mathrm{~m}-\mathrm{o}$ \\
\hline PI225922 & $96.81 \pm 2.11 \mathrm{n}-\mathrm{r}$ & $17.25 \pm 0.43 \mathrm{a}-\mathrm{d}$ & $7.64 \pm 0.18 \mathrm{a}-\mathrm{c}$ & $1466.25 \pm 2.17 \mathrm{a}-\mathrm{i}$ & $58.57 \pm 0.03 \mathrm{~m}-\mathrm{s}$ & $12.33 \pm 1.2 \mathrm{i}-\mathrm{n}$ & $7.54 \pm 0.09 b-f$ & $436.25 \pm 4.09 \mathrm{a}-\mathrm{f}$ \\
\hline PI292894 & $75.37 \pm 2.54 \mathrm{~g}-\mathrm{o}$ & $16.50 \pm 0.87 a-c$ & $10.19 \pm 0.03 f-m$ & $2403.75 \pm 4.09 \mathrm{f}-1$ & $61.07 \pm 1.99 \mathrm{n}-\mathrm{t}$ & $15 \pm 0.58 \mathrm{~m}-\mathrm{p}$ & $9.97 \pm 0.4 \mathrm{i}-\mathrm{o}$ & $1639.17 \pm 162.14 \mathrm{k}-\mathrm{n}$ \\
\hline PI292899 & $100.73 \pm 2.56 \mathrm{p}-\mathrm{t}$ & $23.25 \pm 1.30 \mathrm{e}-\mathrm{i}$ & $9.18 \pm 0.01 b-i$ & $3641.94 \pm 1121.641-\mathrm{q}$ & $56.83 \pm 0.36 \mathrm{k}-\mathrm{s}$ & $4.5 \pm 0.29 \mathrm{a}-\mathrm{e}$ & $13.1 \pm 0.12 \mathrm{tu}$ & $1756.81 \pm 773.881-n$ \\
\hline PI293468 & $81.27 \pm 7.66 h-p$ & $14.25 \pm 0.43 a$ & $10.01 \pm 0.09 \mathrm{e}-\mathrm{m}$ & $1032.5 \pm 101.52 \mathrm{a}-\mathrm{f}$ & $49.13 \pm 2.21 \mathrm{~g}-\mathrm{p}$ & $6.00 \pm 0.00 \mathrm{c}-\mathrm{g}$ & $11.11 \pm 0.03 n-r$ & $562.50 \pm 7.22 c-f$ \\
\hline PI304150 & $90.84 \pm 5.22 \mathrm{k}-\mathrm{q}$ & $31.83 \pm 1.591-n$ & $11.12 \pm 0.36 \mathrm{i}-\mathrm{o}$ & $4993.75 \pm 157.09 \mathrm{q}-\mathrm{t}$ & $107.9 \pm 8.15 x$ & $26.42 \pm 0.68 \mathrm{t}$ & $10.74 \pm 0.25 n-q$ & $3950.42 \pm 957.68 \mathrm{uv}$ \\
\hline PI339600 & $87.83 \pm 5.11 \mathrm{j}-\mathrm{q}$ & $40.50 \pm 6.06 \mathrm{op}$ & $9.36 \pm 0.04 c-j$ & $5473.33 \pm 620.17 \mathrm{st}$ & $71.24 \pm 12.08 \mathrm{r}-\mathrm{v}$ & $12.5 \pm 0.29 \mathrm{j}-\mathrm{n}$ & $9.66 \pm 0.02 \mathrm{~g}-\mathrm{n}$ & $1974.17 \pm 403.66$ no \\
\hline PI339607 & $81.46 \pm 10.61 \mathrm{~h}-\mathrm{p}$ & $32.25 \pm 0.431-n$ & $8.74 \pm 1.47 \mathrm{a}-\mathrm{g}$ & $3930.42 \pm 348.09 \mathrm{~m}-\mathrm{r}$ & $44.91 \pm 3.41 \mathrm{~g}-\mathrm{o}$ & $5.5 \pm 0.87 b-f$ & $8.35 \pm 0.40 c-h$ & $977.08 \pm 456.83 \mathrm{hi}$ \\
\hline PI354466 & $108.21 \pm 7.80 \mathrm{q}-\mathrm{t}$ & $22.5 \pm 1.73 \mathrm{~d}-\mathrm{h}$ & $11.81 \pm 0.841-\mathrm{o}$ & $2440.83 \pm 1144.12 \mathrm{~g}-1$ & $89.03 \pm 3.34 w$ & $8.5 \pm 2.02 \mathrm{e}-\mathrm{j}$ & $10.46 \pm 0.55 \mathrm{~m}-\mathrm{q}$ & $1205.83 \pm 203.03 \mathrm{ij}$ \\
\hline PI354864 & $37.91 \pm 0.05 \mathrm{a}-\mathrm{d}$ & $24.75 \pm 1.30 f-j$ & $9.8 \pm 1.09 \mathrm{~d}-1$ & $1109.17 \pm 85.16 \mathrm{a}-\mathrm{g}$ & $55.17 \pm 7.21 \mathrm{j}-\mathrm{r}$ & $7.5 \pm 0.29 \mathrm{e}-\mathrm{h}$ & $9.84 \pm 0.33 \mathrm{~h}-\mathrm{n}$ & $200.56 \pm 45.16 \mathrm{ab}$ \\
\hline PI358716 & $35.86 \pm 1.89 \mathrm{a}-\mathrm{c}$ & $21.00 \pm 0.00 \mathrm{~b}-\mathrm{g}$ & $11.59 \pm 0.16 \mathrm{k}-\mathrm{o}$ & $2562.92 \pm 273.52 \mathrm{i}-\mathrm{m}$ & $56.23 \pm 4.96 \mathrm{k}-\mathrm{r}$ & $19.5 \pm 0.29 q-s$ & $4.94 \pm 0.17 \mathrm{a}$ & $952.08 \pm 39.21 \mathrm{~g}-\mathrm{i}$ \\
\hline PI487518 & $66.55 \pm 0.26 f-j$ & $16.50 \pm 0.87 a-c$ & $9.32 \pm 0.09 c-i$ & $929.58 \pm 13.23 \mathrm{abcd}$ & $56.55 \pm 0.26 \mathrm{k}-\mathrm{s}$ & $16 \pm 2.89 n-s$ & $8.94 \pm 0.02 \mathrm{f}-\mathrm{m}$ & $235.42 \pm 2.17 \mathrm{a}-\mathrm{c}$ \\
\hline PI527263 & $110.17 \pm 18.12 \mathrm{q}-\mathrm{t}$ & $18.00 \pm 0.87 \mathrm{a}-\mathrm{e}$ & $10.52 \pm 0.02 \mathrm{~g}-\mathrm{m}$ & $2979.17 \pm 157.33 j-n$ & $68.96 \pm 1.94 \mathrm{q}-\mathrm{u}$ & $16.5 \pm 0.29 n-s$ & $11.16 \pm 0.16 n-r$ & $2124.17 \pm 90.45 o p$ \\
\hline PI527302 & $118.97 \pm 9.65 \mathrm{r}-\mathrm{u}$ & $24.67 \pm 1.09 f-j$ & $11.34 \pm 0.28 \mathrm{j}-\mathrm{o}$ & $5127.22 \pm 537.19$ rst & $60.3 \pm 5.14 n-t$ & $20.33 \pm 1.09 \mathrm{~s}$ & $11.9 \pm 0.25 \mathrm{q}-\mathrm{t}$ & $4195.28 \pm 220.4 \mathrm{v}$ \\
\hline PI527561 & $54 \pm 12.12 \mathrm{~b}-\mathrm{g}$ & $29.25 \pm 4.76 \mathrm{j}-\mathrm{m}$ & $9.99 \pm 0.27 \mathrm{e}-\mathrm{m}$ & $7345.28 \pm 394.64 u$ & $125.28 \pm 12.38 \mathrm{x}$ & $11 \pm 1.15 \mathrm{~h}-\mathrm{m}$ & $11.82 \pm 0.04 \mathrm{p}-\mathrm{t}$ & $4970.97 \pm 550.07 w$ \\
\hline PI582531 & $54.89 \pm 0.06 \mathrm{c}-\mathrm{g}$ & $29.25 \pm 3.90 \mathrm{j}-\mathrm{m}$ & $8.61 \pm 0.04 a-g$ & $1161.67 \pm 168.39 a-h$ & $23.66 \pm 0.2 \mathrm{a}-\mathrm{e}$ & $18.5 \pm 3.75 \mathrm{p}-\mathrm{s}$ & $8.97 \pm 0.07 \mathrm{f}-\mathrm{m}$ & $962.08 \pm 0.72 \mathrm{~g}-\mathrm{i}$ \\
\hline PI582581 & $50.02 \pm 2.5 b-f$ & $25.50 \pm 0.87 \mathrm{f}-\mathrm{k}$ & $9.33 \pm 0.59 c-j$ & $4015.83 \pm 97.67 n-r$ & $19.21 \pm 0.92 \mathrm{a}-\mathrm{c}$ & $8.00 \pm 0.58 \mathrm{e}-\mathrm{i}$ & $10.88 \pm 0.12 \mathrm{n}-\mathrm{r}$ & $678.75 \pm 98.39 \mathrm{e}-\mathrm{h}$ \\
\hline PI582697 & $74.29 \pm 15.12 \mathrm{~g}-\mathrm{n}$ & $24.75 \pm 0.43 \mathrm{f}-\mathrm{j}$ & $7.12 \pm 0.1 \mathrm{a}$ & $3388.33 \pm 325.24 j-p$ & $37.97 \pm 2.93 \mathrm{~d}-\mathrm{j}$ & $16 \pm 1.53 n-s$ & $7.06 \pm 0.52 b-d$ & $2386.67 \pm 637.49 \mathrm{pq}$ \\
\hline PI582707 & $122.81 \pm 7.4 \mathrm{tu}$ & $30.75 \pm 0.43 \mathrm{k}-\mathrm{m}$ & $8.93 \pm 0.18 a-h$ & $5937.64 \pm 349.33 \mathrm{t}$ & $52.58 \pm 12.65 \mathrm{i}-\mathrm{q}$ & $13 \pm 1.73 \mathrm{k}-\mathrm{n}$ & $8.86 \pm 0.61 f-1$ & $3659.44 \pm 1073.66 \mathrm{u}$ \\
\hline PI582785 & $68.75 \pm 0.72 \mathrm{f}-1$ & $36.75 \pm 0.43 \mathrm{no}$ & $9.41 \pm 0 c-j$ & $840.00 \pm 11.07 \mathrm{a}-\mathrm{d}$ & $40.98 \pm 0.01 \mathrm{e}-1$ & $12.5 \pm 0.87 \mathrm{j}-\mathrm{n}$ & $11.84 \pm 0.03 p-t$ & $756.25 \pm 2.17 f-h$ \\
\hline
\end{tabular}




\section{Continued}

\begin{tabular}{|c|c|c|c|c|c|c|c|c|}
\hline PI582789 & $63.73 \pm 12.86 \mathrm{e}-\mathrm{i}$ & $42.75 \pm 3.90 p$ & $8.75 \pm 0.39 \mathrm{a}-\mathrm{g}$ & $3416.67 \pm 626.43 \mathrm{k}-\mathrm{p}$ & $51.84 \pm 0.48 \mathrm{hi}-\mathrm{q}$ & $19.5 \pm 4.33 \mathrm{q}-\mathrm{s}$ & $8.26 \pm 0.07 \mathrm{~b}-\mathrm{g}$ & $1704.17 \pm 388.751-n$ \\
\hline PI582793 & $91.53 \pm 4.55 \mathrm{l}-\mathrm{q}$ & $16.25 \pm 0.90 \mathrm{a}-\mathrm{c}$ & $12.89 \pm 0.64 n-p$ & $3025.83 \pm 726.70 \mathrm{j}-\mathrm{n}$ & $50.56 \pm 10.62 \mathrm{hi}-\mathrm{p}$ & $10 \pm 1.73 \mathrm{~g}-1$ & $10.35 \pm 1.22 \mathrm{l}-\mathrm{p}$ & $1803.75 \pm 735.07 \mathrm{l}-\mathrm{o}$ \\
\hline PI582853 & $83.99 \pm 2.08 \mathrm{~h}-\mathrm{p}$ & $22.50 \pm 3.46 \mathrm{~d}-\mathrm{h}$ & $16.59 \pm 0.24 \mathrm{rs}$ & $2490.42 \pm 16.60 \mathrm{~g}-1$ & $77.33 \pm 1.46 \mathrm{tu}-\mathrm{w}$ & $15.67 \pm 0.33 n-r$ & $13.09 \pm 0.05 \mathrm{tu}$ & $1487.92 \pm 365.51 j-1$ \\
\hline PI582867 & $120.93 \pm 3.93 \mathrm{~s}-\mathrm{u}$ & $23.25 \pm 0.43 \mathrm{e}-\mathrm{i}$ & $14.2 \pm 0.09 \mathrm{pq}$ & $4448.47 \pm 585.01 \mathrm{o}-\mathrm{s}$ & $88.47 \pm 6.65 \mathrm{vw}$ & $9.00 \pm 1.15 f-k$ & $13.99 \pm 0.45 \mathrm{uv}$ & $2981.53 \pm 720.11 \mathrm{st}$ \\
\hline PI583182 & $89.1 \pm 3.58 \mathrm{j}-\mathrm{q}$ & $24.75 \pm 0.43 f-j$ & $9.78 \pm 0.44 \mathrm{~d}-\mathrm{k}$ & $2885.00 \pm 353.15 j-n$ & $60.41 \pm 9.81 \mathrm{n}-\mathrm{t}$ & $7.5 \pm 1.44 \mathrm{e}-\mathrm{h}$ & $10.03 \pm 0.31 \mathrm{j}-\mathrm{o}$ & $1525.83 \pm 72.17 \mathrm{j}-1$ \\
\hline PI583205 & $53.6 \pm 1.69 \mathrm{~b}-\mathrm{g}$ & $27.75 \pm 0.43 \mathrm{~h}-\mathrm{m}$ & $8.67 \pm 0.16 \mathrm{a}-\mathrm{g}$ & $4648.19 \pm 263.89 \mathrm{p}-\mathrm{t}$ & $35.41 \pm 1.36 \mathrm{c}-\mathrm{i}$ & $13.5 \pm 2.02 \mathrm{l}-\mathrm{o}$ & $8.49 \pm 0.38 \mathrm{~d}-\mathrm{i}$ & $3934.72 \pm 189.29 u v$ \\
\hline PI583209 & $83.82 \pm 35.56 h-p$ & $29.25 \pm 3.03 \mathrm{j}-\mathrm{m}$ & $10.44 \pm 0.22 \mathrm{~g}-\mathrm{m}$ & $2019.44 \pm 291.98 b-j$ & $52.89 \pm 0.06 \mathrm{j}-\mathrm{q}$ & $18.33 \pm 2.03 p-s$ & $9 \pm 0.33 \mathrm{f}-\mathrm{m}$ & $1293.47 \pm 217.31 \mathrm{i}-\mathrm{k}$ \\
\hline PI583254 & $108.01 \pm 17.88 \mathrm{q}-\mathrm{t}$ & $12.75 \pm 0.43 a$ & $8.13 \pm 0.18 \mathrm{a}-\mathrm{e}$ & $633.75 \pm 163.82 \mathrm{ab}$ & $61.81 \pm 17.810-\mathrm{t}$ & $1.00 \pm 0.00 \mathrm{a}$ & $10.22 \pm 0.01 \mathrm{k}-\mathrm{o}$ & $247.5 \pm 60.14 a-c$ \\
\hline PI610520 & $86.73 \pm 0.71 \mathrm{i}-\mathrm{q}$ & $33.00 \pm 4.33 \mathrm{mn}$ & $16.16 \pm 3.24 \mathrm{qr}$ & $4553.33 \pm 667.8 \mathrm{pq}-\mathrm{t}$ & $64.27 \pm 10.84 \mathrm{p}-\mathrm{u}$ & $11 \pm 0.58 \mathrm{~h}-\mathrm{m}$ & $10.03 \pm 0.04 j-\mathrm{o}$ & $2980 \pm 331.98 \mathrm{st}$ \\
\hline PI293463 & $163.56 \pm 0.33 \mathrm{v}$ & $30.50 \pm 3.91 \mathrm{k}-\mathrm{m}$ & $7.21 \pm 1.39 \mathrm{ab}$ & $3405.42 \pm 1686.58 j-p$ & $80.96 \pm 1.26 \mathrm{u}-\mathrm{w}$ & $9.5 \pm 3.75 f-1$ & $8.5 \pm 0.43 \mathrm{~d}-\mathrm{j}$ & $629.58 \pm 231.66 \mathrm{~d}-\mathrm{h}$ \\
\hline PI339598 & $69.56 \pm 4.27 \mathrm{f}-1$ & $29.75 \pm 0.90 \mathrm{j}-\mathrm{m}$ & $8.56 \pm 0.81 \mathrm{a}-\mathrm{g}$ & $2520.00 \pm 505.77 \mathrm{~h}-1$ & $42.2 \pm 9.48 \mathrm{f}-\mathrm{m}$ & $15 \pm 0.58 \mathrm{~m}-\mathrm{p}$ & $6.85 \pm 0.31 b c$ & $1135.83 \pm 253.38 \mathrm{i}$ \\
\hline PI632777 & $82.09 \pm 10.02 \mathrm{~h}-\mathrm{p}$ & $21.33 \pm 1.45 \mathrm{c}-\mathrm{g}$ & $11.97 \pm 0.17 \mathrm{~m}-\mathrm{o}$ & $4481.67 \pm 675.85 \mathrm{o}-\mathrm{s}$ & $57.15 \pm 7.741-\mathrm{s}$ & $13.17 \pm 2.89 \mathrm{k}-\mathrm{n}$ & $17.29 \pm 0.03 x$ & $3269.17 \pm 933.17 t$ \\
\hline PI632796 & $49.02 \pm 0.06 b-f$ & $23.25 \pm 0.43 \mathrm{e}-\mathrm{i}$ & $8.17 \pm 0.01 \mathrm{a}-\mathrm{e}$ & $2185.00 \pm 105.32 \mathrm{~d}-\mathrm{k}$ & $34.61 \pm 1.65 b-h$ & $19.67 \pm 3.76 \mathrm{rs}$ & $8.54 \pm 0.07 \mathrm{~d}-\mathrm{j}$ & $1682.5 \pm 246.661-n$ \\
\hline PI663009 & $60.46 \pm 1.29 \mathrm{~d}-\mathrm{h}$ & $15.75 \pm 2.17 \mathrm{ab}$ & $11.09 \pm 0.05 \mathrm{i}-\mathrm{o}$ & $951.39 \pm 256.94 \mathrm{a}-\mathrm{e}$ & $51.99 \pm 2.47 \mathrm{~h}-\mathrm{q}$ & $4.33 \pm 0.88 \mathrm{a}-\mathrm{e}$ & $6.79 \pm 0.76 b$ & $509.44 \pm 353.5 b-f$ \\
\hline SARI-6-2-6 & $67.78 \pm 12.82 \mathrm{f}-\mathrm{k}$ & $23.25 \pm 0.43 \mathrm{e}-\mathrm{i}$ & $18.58 \pm 0.24 \mathrm{st}$ & $3103.33 \pm 509.31 \mathrm{j}-\mathrm{o}$ & $40.57 \pm 8.59 \mathrm{e}-1$ & $13.83 \pm 1.691-o$ & $16.54 \pm 0.45 w x$ & $2497.5 \pm 558.2 \mathrm{qr}$ \\
\hline SONGOTRA & $72.28 \pm 5.92 \mathrm{f}-\mathrm{m}$ & $26.25 \pm 3.03 \mathrm{~g}-\mathrm{k}$ & $16.11 \pm 0.58 \mathrm{qr}$ & $717.5 \pm 184.75 \mathrm{ab}$ & $43.67 \pm 5.55 \mathrm{~g}-\mathrm{n}$ & $6.5 \pm 0.29 c-g$ & $15.11 \pm 0.38 \mathrm{vw}$ & $406.67 \pm 16.84 a-f$ \\
\hline WANGKAE & $138.34 \pm 14.77 \mathrm{u}$ & $15.75 \pm 0.43 \mathrm{ab}$ & $19.78 \pm 0.03 \mathrm{tu}$ & $544.17 \pm 1.44 \mathrm{a}$ & $61.84 \pm 17.33 \mathrm{o}-\mathrm{t}$ & $3.00 \pm 0.00 \mathrm{a}-\mathrm{d}$ & $14.82 \pm 0.46 \mathrm{v}$ & $288.75 \pm 62.31 \mathrm{a}-\mathrm{d}$ \\
\hline
\end{tabular}

DSW = Dry shoot weight, NPplt $=$ Number of pods per plant, HSW = Hundred seed weight and YLDha $=$ Grain yield per hectare.

Table 4. Drought indices of 50 cowpea genotypes grown under drought stress (DS) and non-stress (NS) conditions at the Bontanga site in Northern Ghana.

\begin{tabular}{ccccccccccc}
\hline Genotype & NS & DS & DSI & DTI & MP & GMP & YI & YRR & YSI \\
\hline PI582785 & 840 & 756 & 0.18 & 0.90 & 798.00 & 796.89 & 0.5 & 0.10 & 0.90 \\
PI583205 & 4648 & 3935 & 0.27 & 0.85 & 4291.50 & 4276.67 & 2.6 & 0.15 & 0.85 \\
PI582531 & 1162 & 962 & 0.30 & 0.83 & 1062.00 & 1057.28 & 0.6 & 0.17 & 0.83 & 0.82 \\
PI527302 & 5127 & 4195 & 0.32 & 0.82 & 4661.00 & 4637.65 & 2.8 & 0.18 & 0.19 & 0.81 \\
PI221732 & 2343 & 1891 & 0.34 & 0.81 & 2117.00 & 2104.90 & 1.3 & 0.19 & 0.81 \\
SARI-6-2-6 & 3103 & 2498 & 0.34 & 0.81 & 2800.50 & 2784.11 & 1.7 & 0.19 \\
PI194207 & 781 & 620 & 0.36 & 0.79 & 700.50 & 695.86 & 0.4 & 0.21 & 0.79 \\
PI304150 & 4994 & 3950 & 0.37 & 0.79 & 4472.00 & 4441.43 & 2.6 & 0.21 & 0.79 \\
PADITUYA & 2154 & 1695 & 0.37 & 0.79 & 1924.50 & 1910.77 & 1.1 & 0.21 & 0.79 \\
PI632796 & 2185 & 1683 & 0.40 & 0.77 & 1934.00 & 1917.64 & 1.1 & 0.23 & 0.77 \\
PI200867 & 3693 & 2838 & 0.41 & 0.77 & 3265.50 & 3237.40 & 1.9 & 0.23 & 0.77 \\
PI632777 & 4482 & 3269 & 0.47 & 0.73 & 3875.50 & 3827.75 & 2.2 & 0.27 & 0.73 \\
PI293470 & 390 & 283 & 0.48 & 0.73 & 336.50 & 332.22 & 0.2 & 0.27 & 0.73 \\
PI527263 & 2979 & 2124 & 0.50 & 0.71 & 2551.50 & 2515.43 & 1.4 & 0.29 & 0.71 \\
PI582697 & 3388 & 2387 & 0.52 & 0.70 & 2887.50 & 2843.79 & 1.6 & 0.30 & 0.70 \\
\hline
\end{tabular}




\section{Continued}

\begin{tabular}{|c|c|c|c|c|c|c|c|c|c|}
\hline PI292894 & 2404 & 1639 & 0.56 & 0.68 & 2021.50 & 1984.98 & 1.1 & 0.32 & 0.68 \\
\hline PI527561 & 7345 & 4971 & 0.57 & 0.68 & 6158.00 & 6042.52 & 3.3 & 0.32 & 0.68 \\
\hline PI582867 & 4448 & 2982 & 0.58 & 0.67 & 3715.00 & 3641.97 & 2.0 & 0.33 & 0.67 \\
\hline PI610520 & 4553 & 2980 & 0.61 & 0.65 & 3766.50 & 3683.47 & 2.0 & 0.35 & 0.65 \\
\hline PI194212 & 772 & 499 & 0.62 & 0.65 & 635.50 & 620.67 & 0.3 & 0.35 & 0.65 \\
\hline PI583209 & 2019 & 1293 & 0.63 & 0.64 & 1656.00 & 1615.72 & 0.9 & 0.36 & 0.64 \\
\hline PI165486 & 677 & 431 & 0.64 & 0.64 & 554.00 & 540.17 & 0.3 & 0.36 & 0.64 \\
\hline PI582707 & 5938 & 3659 & 0.67 & 0.62 & 4798.50 & 4661.24 & 2.4 & 0.38 & 0.62 \\
\hline IT86D-610 & 795 & 487 & 0.68 & 0.61 & 641.00 & 622.23 & 0.3 & 0.39 & 0.61 \\
\hline PI582853 & 2490 & 1488 & 0.71 & 0.60 & 1989.00 & 1924.87 & 1.0 & 0.40 & 0.60 \\
\hline PI582793 & 3026 & 1804 & 0.71 & 0.60 & 2415.00 & 2336.43 & 1.2 & 0.40 & 0.60 \\
\hline SONGOTRA & 718 & 407 & 0.76 & 0.57 & 562.50 & 540.58 & 0.3 & 0.43 & 0.57 \\
\hline PI293468 & 1033 & 563 & 0.80 & 0.55 & 798.00 & 762.61 & 0.4 & 0.45 & 0.55 \\
\hline PI663009 & 951 & 509 & 0.82 & 0.54 & 730.00 & 695.74 & 0.3 & 0.46 & 0.54 \\
\hline WANGKAE & 544 & 289 & 0.82 & 0.53 & 416.50 & 396.50 & 0.2 & 0.47 & 0.53 \\
\hline PI583182 & 2885 & 1526 & 0.83 & 0.53 & 2205.50 & 2098.22 & 1.0 & 0.47 & 0.53 \\
\hline PI582789 & 3417 & 1704 & 0.88 & 0.50 & 2560.50 & 2413.00 & 1.1 & 0.50 & 0.50 \\
\hline PI354466 & 2441 & 1206 & 0.89 & 0.49 & 1823.50 & 1715.76 & 0.8 & 0.51 & 0.49 \\
\hline PI214354 & 3253 & 1578 & 0.90 & 0.49 & 2415.50 & 2265.66 & 1.0 & 0.51 & 0.49 \\
\hline PI292899 & 3642 & 1757 & 0.91 & 0.48 & 2699.50 & 2529.62 & 1.2 & 0.52 & 0.48 \\
\hline ITOK-837-1 & 754 & 349 & 0.94 & 0.46 & 551.50 & 512.98 & 0.2 & 0.54 & 0.46 \\
\hline PI339598 & 2520 & 1136 & 0.96 & 0.45 & 1828.00 & 1691.96 & 0.8 & 0.55 & 0.45 \\
\hline PI205141 & 4623 & 1933 & 1.02 & 0.42 & 3278.00 & 2989.36 & 1.3 & 0.58 & 0.42 \\
\hline PI583254 & 634 & 248 & 1.07 & 0.39 & 441.00 & 396.52 & 0.2 & 0.61 & 0.39 \\
\hline PI358716 & 2563 & 952 & 1.10 & 0.37 & 1757.50 & 1562.04 & 0.6 & 0.63 & 0.37 \\
\hline PI339600 & 5473 & 1974 & 1.12 & 0.36 & 3723.50 & 3286.90 & 1.3 & 0.64 & 0.36 \\
\hline PI225922 & 1466 & 436 & 1.23 & 0.30 & 951.00 & 799.48 & 0.3 & 0.70 & 0.30 \\
\hline PI186460 & 513 & 146 & 1.26 & 0.28 & 329.50 & 273.67 & 0.1 & 0.72 & 0.28 \\
\hline PI487518 & 930 & 235 & 1.31 & 0.25 & 582.50 & 467.49 & 0.2 & 0.75 & 0.25 \\
\hline PI339607 & 3930 & 977 & 1.32 & 0.25 & 2453.50 & 1959.49 & 0.6 & 0.75 & 0.25 \\
\hline PI194208 & 2479 & 468 & 1.42 & 0.19 & 1473.50 & 1077.11 & 0.3 & 0.81 & 0.19 \\
\hline PI293463 & 3405 & 630 & 1.43 & 0.19 & 2017.50 & 1464.63 & 0.4 & 0.81 & 0.19 \\
\hline PI354864 & 1109 & 201 & 1.44 & 0.18 & 655.00 & 472.13 & 0.1 & 0.82 & 0.18 \\
\hline PI582581 & 4016 & 679 & 1.46 & 0.17 & 2347.50 & 1651.32 & 0.4 & 0.83 & 0.17 \\
\hline ITOHC-303-1 & 3371 & 363 & 1.57 & 0.11 & 1867.00 & 1106.20 & 0.2 & 0.89 & 0.11 \\
\hline Mean & 2668.12 & 1511.7 & 0.7774 & 0.5578 & 2089.91 & 1963.661 & 1.002 & 0.4422 & 0.5578 \\
\hline
\end{tabular}

NS = Non-Drought Stress, DS = Drought stress, DSI = Drought Susceptibility Index, DTI $=$ Drought Tolerant index, MP = Mean Productivity, GMP $=$ Geometric Mean Productivity, YI = Yield Index, YRR = Yield Reduction Rate and YSI = Yield Stability Index. 
Drought susceptibility and tolerance, yield index, yield reduction rate and yield stability index ranked the test genotypes PI582785, PI583205, PI582531, PI527302, PI221732 and SARI-6-2-6 as more drought tolerant than the other genotypes. Even though some genotypes which are considered drought tolerant did not produce the highest yields under non-stressed conditions, they were nonetheless, able to withstand the stress and also produced substantial yields. Genotypes PI225922, PI186460, PI487518, PI339607, PI194208, PI293463, PI354864 and PI582581 were considered susceptible genotypes although some produced high yields under well-watered conditions.

\section{Discussion}

The imposition of the drought stress was effective since the trial was carried out during the dry season and irrigated water could be supplied to the trials in controlled amounts and on a strict time schedule. The daily average temperature was $29.7^{\circ} \mathrm{C}$ which is within favorable range for cowpea growth and development [34], although towards the end of the season maximum temperatures exceeded $33^{\circ} \mathrm{C}$ or more. The soil was sandy loam with $\mathrm{pH}$ of 5.89 which was conducive for cowpea production [34] [35]. However, total rainfall below $15 \mathrm{~mm}$ would not produce a cowpea crop without irrigation which was added to both treatments but in different amounts for NS and DS conditions.

The withdrawal of irrigation water after flower buds' initiation for the DS treatment subjected the crop to moderate to high drought stress. The terminal drought that was experienced in this experiment was typical of the major abiotic stress problem for cowpea production in Sub-Saharan Africa, namely early termination of rains. Short insufficient rains are typical of the Sahelian region of West Africa and sometimes affect the Guinea Savannah region as well and are a major issue for agriculture in Northern Ghana. Terminal drought resulted in poor yield performance of the cowpea plants subjected to the water deficit, which is consistent with the findings of [36] and [37].

The drought stress index of the experiment was 0.57 indicating a moderate to high drought stress subjected to the cowpea genotypes. Variability in the individual values for the genotypes in the trial could be attributed to the genotypic differences and genotype $\mathrm{x}$ environment interactions in the experiment based on how drought affected yielding capacity of each accession. Researches [38] and [39] reported noticeable reduction in the grain yield of cowpea genotypes evaluated under drought condition which was attributed to genotypic differences.

The genotypes showed marked variations in their response to the watering regimes imposed on the cowpeas evaluated in the trial. The significant effect of genotype, watering regime and their interaction on the test cowpeas showed that the genotypes exhibited contrasting responses to the DS versus NS in the present study. Researchers [40] [41] [42] reported variable response of cowpea to drought adaptation which subsequently affected growth and yield. According to [36], the response cowpea can have to drought depends on the genotype, inten- 
sity of the drought as well as the duration of the drought exposure. The genotypes expressed varied response to drought stress based on the measured traits, with the extent of response being more pronounced in some genotypes than others owing to genotypic differences [43].

The significant variation among genotypes in response to drought in this experiment confirmed the findings of [44], that cowpea genotypes will vary in their productivity when exposed to drought at vegetative or reproductive stages. Days to flowering and maturity, hundred seed weight and number of branches were not highly sensitive to drought as their percentage reduction were smaller as compared to other parameters. Dry shoot weight, number of leaves and pods per plant and grain yield were highly sensitive to drought stress. Researchers [45] and [46] reported similar effects of drought on dry matter and yield of cowpea.

The drought stress caused $18.0 \%$ reduction in days taken to reach physiological maturity of cowpea in the current experiment which is similar to the growth period shortening caused by drought in previous reports [47]. The reduction in days to flowering in the current study also confirmed an earlier report by [48]. The variation in days to flowering and maturity among the genotypes in this current trial is a genetic response of those genotypes to water deficit or stress in the soil. Even though the drought stress occurred at the reproductive stage, it affected both flowering and maturity of the cowpea plants.

The genotypes that responded to drought stress through a reduction in the number of days to physiological maturity while maintaining high yields could be linked to drought escape mechanism of the cowpea plants [49].

In this study, some genotypes flowered and matured late and still gave maximum grain yield under drought stressed conditions, suggesting that those genotypes could be linked to drought tolerance [49]. On the other hand, genotypes PI582793, PI582867, SARI-6-2-6, PI583254 and SONGOTRA flowered and reached maturity late under drought stressed conditions. The genotypes PI583209, PI339600, ITOHC-303-1, PI527263 and PI527302 that showed earliness in physiological maturity together with genotypes PI339600, PI527263 and PI527302 produced substantial grain yield under drought conditions, showing that drought escape through early pod set is one mechanism of drought tolerance.

The overall number of pods per plant and grain yield was significantly reduced by the drought stress conditions. Yield component traits are good indicators of drought stress in cowpea [45] [46]. Researcher [48] reported significant reduction of seed yield of cowpea subjected to similar drought stress conditions. The higher reduction in the number of pods per plant under drought stress conditions could be due to flower abortion [50]. Poor carbohydrate partitioning and photosynthate assimilation to the developing seed could be associated with the reduction in 100 seed weight among genotypes in this study [51]. Drought tolerant genotypes have strong association between photosynthate assimilation and carbohydrate accumulation in the developing seed and could maintain high 100 
seed weight regardless of the soil water. Understanding the response of yield component trait to drought could be utilized in plant breeding programs to select or develop superior genotypes with drought tolerance in future. Current breeding programs in SARI, the Savannah Agricultural Research Institute together with IITA, the International Institute for Tropical Agriculture, could benefit from the germplasm evaluated by this study and the results of drought tolerance trait dissection.

\section{Conclusion}

The response of cowpea plants to water deficit depends on genotype, drought intensity, and the growth stage at which drought occurs. In our trial, the number of leaves per plant and dry matter of cowpea were negatively affected by water deficit at flowering, which manifested in up to $50 \%$ yield reduction when compared to well-watered plants. Similarly, the number of days to flowering and maturity were shortened by imposing terminal drought on the test cowpeas in this study. Genotypes PI339600, PI527263, PI527302, PI582793, PI582867 and SARI-6-2-6 have promising yield potentials under drought stress conditions. These genotypes could be exploited for future breeding program that will develop drought tolerant varieties for the savannah ecology of northern Ghana and other areas with similar environmental conditions across West Africa.

\section{Acknowledgements}

We acknowledge the germplasm provided by Dr. Brad Morris of the United States Department of Agriculture (USDA) station in Griffin, Georgia and the cowpea breeding team at SARI headed by Dr. Haruna Mohammed and Dr. Lucas Mackasmiel, TSU for his support. The work is supported by Borlaug Higher Education for Agriculture Research Development (BHEARD) under United States Agency for International Development (USAID) as part of the Feed the Future initiative. We acknowledged support from Savannah Agriculture Research Institute (SARI), Tennessee State University (TSU) and the University for Development Studies (UDS). The Evans Allen grant (TEN-X) to TSU is recognized for funding the senior authors work on this project.

\section{Conflicts of Interest}

The authors declare that there is no conflict of interest.

\section{References}

[1] Kamara, A.Y., Omoigui, L.O., Kamai, N., Ewansiha, S.U. and Ajeigbe, H.A. (2018) Improving Cultivation of Cowpea in West Africa. In: Sivasankar, S., et al., Eds., Achieving Sustainable Cultivation of Grain Legumes, Burleigh Dodds Science Publishing Limited, Volume 2, 235-252. https://doi.org/10.19103/AS.2017.0023.30

[2] Inaizumi, H., Singh, B.B., Sanginga, P.C., Manyong, V.M., Adesina, A.A. and Tarawali, S. (1999) Adoption and Impact of Dry-Season Dual-Purpose Cowpea in the 
Semiarid Zone of Nigeria (IITA IMPAC). IITA and Meg-Comm Network, Ibadan, Nigeria.

[3] Hall, A.E. (2012) Phenotyping Cowpeas for Adaptation to Drought. Frontiers in Physiology, 3, 1-8. https://doi.org/10.3389/fphys.2012.00155

[4] Egbe, O., Alibo, S. and Nwueze, I. (2010) Evaluation of Some Extra-Early and Early-Maturing Cowpea Varieties for Intercropping with Maize in Southern Guinea Savanna of Nigeria. Agriculture and Biology Journal of North America, 1, 845-858. https://doi.org/10.5251/abjna.2010.1.5.845.858

[5] Gomez Carlos, P. (2004) Cowpea Post-Harvest Operations. Food and Agriculture Organization of the United Nations, 1-70. http://www.fao.org/3/a-au994e.pdf

[6] Singh, B.B. (2014) Future Prospects of Cowpea. In: The Food Legume of the $21 \mathrm{st}$ Century, Crop Science Society of America, Madison, 145-157. https://doi.org/10.2135/2014.cowpea.c7

[7] Tony, N., James, O. and Nixon, T. (2015) Cowpea Production Handbook. IFS/AGRA, Juba.

[8] Singh, B.B. (2014) Origin, Distribution, and Importance. In: The Food Legume of the 21 st Century, Crop Science Society of America, Madison, 1-15.

[9] Timko, M.P. and Singh, B.B. (2008) Cowpea, a Multifunctional Legume. In: Moore P.H. and Ming, R., Eds., Genomics of Tropical Crop Plants, Springer, New York, 227-258. https://doi.org/10.1007/978-0-387-71219-2_10

[10] MoFA/SRID (2011) Agriculture in Ghana. Facts and Figures, Accra.

[11] Egbadzor, K.F., Yeboah, M., Offei, S.K., Ofori K. and Danquah, E.Y. (2013) Farmers' Key Production Constraints and Traits Desired in Cowpea in Ghana. Journal of Agricultural Extension and Rural Development, 5, 14-20.

[12] MoFA (2016) Agriculture in Ghana, Facts and Figures. Ministry of Food and Agriculture, Statistics, Research and Information Directorate (SRID), 25, 1-113. https://www.agrofood-westafrica.com/fileadmin/user_upload/messen/agrofood-We stafrica/Brochure/Agriculture-in-Ghana-Facts-and-Figures-2015.pdf

[13] Singh, B.B. (2014) Production Constraints. In: The Food Legume of the 21 st Century, Crop Science Society of America, Madison, 35-53. https://doi.org/10.2135/2014.cowpea.c3

[14] Lesk, C., Rowhani, P. and Ramankutty, N. (2016) Influence of Extreme Weather Disasters on Global Crop Production. Nature, 529, 84-87. https://doi.org/10.1038/nature16467

[15] Fahad, S., Bajwa, A.A., Nazir, U., Anjum, S.A., Farooq, A., Zohaib, A., Sadia, S., Nasim, W., Adkins, S., Saud, S., Ihsan, M.Z., Alharby, H., Wu, C., Wang, D. and Huang, J. (2017) Crop Production under Drought and Heat Stress: Plant Responses and Management Options. Frontiers in Plant Science, 8, 1147.

[16] Barnabás, B., Jäger, K. and Fehér, A. (2008) The Effect of Drought and Heat Stress on Reproductive Processes in Cereals. Plant, Cell and Environment, 31, 11-38.

[17] Dellal, I. and McCarl, B.A. (2010) The Economic Impacts of Drought on Agriculture: The Case of Turkey. In: López-Francos, A. and López-Francos, A., Eds., Economics of Drought and Drought Preparedness in a Climate Change Context, CIHEAM/FAO/ICARDA/GDAR/CEIGRAM/MARM, Zaragoza, CIHEAM Options Méditerranéennes, No. 95, 169-174. http://ressources.ciheam.org/om/pdf/a95/00801342.pdf

[18] Jaleel, C.A., Manivannan, P., Abdul Wahid, M.F., Al-Juburi, H.J., Somasundaram, 
R. and Panneerselvam, R. (2009) Drought Stress in Plants: A Review on Morphological Characteristics and Pigments Composition. International Journal of Agriculture and Biology, 11, 100-105.

[19] Rehman, M.U., Rather, G.H., Gull, Y., Mir, M.R. Mir, M.M., Waida, U.I. and Hakeem, K.R. (2015) Effect of Climate Change on Horticultural. In: Hakeem, K.R., Ed., Crop Production and Global Environment Issues, Springer, Berlin, 211-239. https://doi.org/10.1007/978-3-319-23162-4_9

[20] Eziz, A., Yan, Z., Tian, D., Han, W., Tang, Z. and Fang, J. (2017) Drought Effect on Plant Biomass Allocation: A Meta-Analysis. Ecology and Evolution, 7, 11002-11010. https://doi.org/10.1002/ece3.3630

[21] Neumann, P.M. (2008) Coping Mechanisms for Crop Plants in Drought-Prone Environments. Annals of Botany, 101, 901-907. https://doi.org/10.1093/aob/mcn018

[22] Van Loon, A.F. (2015) Hydrological Drought Explained. WIREs Water, 2, 359-392. https://doi.org/10.1002/wat2.1085

[23] Farrell, D., Trotman, A. and Cox, C. (2011) Global Assessment Report on Disaster Risk Reduction: Drought Early Warning and Risk Reduction. A Case Study of the Caribbean Drought of 2009-2010. International Federation of the Red Cross.

[24] Kumar, D. and Narain, P. (2005) Production Technology for Cowpea. ACIRP on Arid Legumes, CAZRI. Jodhpur, Rajasthan.

[25] SARI (2015) Savvannah Agriculture Research Institute 2015 Metrological Department Report.

[26] Walkley, A.J. and Black, I.A. (1934) Estimation of Soil Organic Carbon by the Chromic Acid Titration Method. Soil Science, 37, 29-38.

https://doi.org/10.1097/00010694-193401000-00003

[27] Bray, R.H. and Kurtz, L.T. (1945) Determination of Total Organic and Available Forms of Phosphorus in Soils. Soil Science, 59, 39-45. https://doi.org/10.1097/00010694-194501000-00006

[28] Toth, S.J. and Prince, A.L. (1949) Estimation of Cation-Exchange Capacity and Exchangeable $\mathrm{Ca}, \mathrm{K}$, and Na Contents of Soils by Flame Photometer Techniques. Soil Science, 67, 439-446. https://doi.org/10.1097/00010694-194906000-00003

[29] Fischer, R.A. and Maurer, R. (1978) Drought Resistance in Spring Wheat Cultivars. I Grain Yield Responses. Australian Journal of Agricultural Research, 29, 897-912. https://doi.org/10.1071/AR9780897

[30] Fernandez, G.C.J. (1992) Effective Selection Criteria for Assessing Stress Tolerance. In: Kuo, C.G.E., Ed., Proceedings of the International Symposium on Adaptation of Vegetables and Other Food Crops in Temperature and Water Stress, AVRDC Publication, Tainan, 257-270.

[31] Rosielle, A.A. and Hamblin, J. (1981) Theoretical Aspects of Selection for Yield in Stress and Non-Stress Environment. Crop Science, 21, 943-946. https://doi.org/10.2135/cropsci1981.0011183X002100060033x

[32] Gavuzzi, P., Rizza, F., Palumbo, M., Campanille, R., Ricciardi, G. and Borghi, B. (1997) Evaluation of Field and Laboratory Predictors of Drought and Heat Tolerance in Winter Cereals. Canadian Journal of Plant Science, 77, 523-531. https://doi.org/10.4141/P96-130

[33] Bouslama, M. and Schapaugh Jr., W.T. (1984) Stress Tolerance in Soybeans. I. Evaluation of Three Screening Techniques for Heat and Drought Tolerance. Crop Science, 24, 933-937. https://doi.org/10.2135/cropsci1984.0011183X002400050026x 
[34] DPP (2014) Production Guidelines for Cowpea. Pretoria. http://www.daff.gov.za

[35] Davis, D.W., Oelke, E.A., Oplinger, E.S., Doll, J.D., Hanson, C.V. and Putnam, D.H. (1991) Alternative Field Crops Manual: Crambe. University of Wisconsin, Madison. https://www.hort.purdue.edu/newcrop/afcm/crambe.html

[36] Pejić, B., Mačkić, K., Mikić, A., Ćupina, B., Peksen, E. and Antanasović, S. (2013) Effect of Water Stress on the Yield of Cowpea (Vigna unguiculata L. Walp.) in Temperate Climatic Conditions. Contemporary Agriculture/ Savremena Poljoprivreda, 62, 168-176.

[37] Shao, H.B., Chu, L.Y., Jaleel, C.A. and Zhao, C.X. (2008) Water-Deficit Stress-Induced Anatomical Changes in Higher Plants. Comptes Rendus-Biologies, 331, 215-225. https://doi.org/10.1016/j.crvi.2008.01.002

[38] Ishiyaku, M.F. and Aliyu, H. (2013) Field Evaluation of Cowpea Genotypes for Drought Tolerance and Striga Resistance in the Dry Savanna of the North-West Nigeria-SciAlert Responsive Version. International Journal of Plant Breeding and Genetics, 7, 47-56. https://doi.org/10.3923/ijpbg.2013.47.56

[39] Mwale, S.E., Ochwo-ssemakula, M., Sadik, K., Achola, E., Okul, V., Gibson, P. and Rubaihayo, P. (2017) Response of Cowpea Genotypes to Drought Stress in Uganda. American Journal of Plant Science, 8, 720-733. https://doi.org/10.4236/ajps.2017.84050

[40] Turk, K.J. and Hall, A.E. (1980) Drought Adaptation of Cowpea. IV. Influence of Drought on Water Use, and Relations with Growth and Seed Yield. Agronomy Journal, 72, 434-439. https://doi.org/10.2134/agronj1980.00021962007200030007x

[41] Turk, K.J., Hall, A.E. and Asbel, C.W. (1980) Drought Adaptation of Cowpea (I) Influence of Drought on seed Yield. Agronomy Journal, 72, 413-420. https://doi.org/10.2134/agronj1980.00021962007200030004x

[42] Farooq, M., Gogoi, N., Barthakur, S., Baroowa, B., Bharadwaj, N., Alghamdi, S.S. and Siddique, K.H.M. (2016) Drought Stress in Grain Legumes during Reproduction and Grain Filling. Journal of Agronomy and Crop Science, 1-22.

[43] Ajayi, A.T., Gbadamosi, A.E. and Olumekun, V.O. (2018) Screening for Drought Tolerance in Cowpea (Vigna unguiculata L. Walp) at Seedling Stage under Screen House Condition. International Journal of Biosciences and Technology, 11, 1-19.

[44] Qasem, J.R. and Biftu, K.N. (2010) Growth Analysis and Responses of Cowpea (Vigna sinensis L. Savi Ex Hassk.) and Redroot Pigweed (Amaranthus retroflexus L.), Grown in Pure and Mixed Stands, to Density and Water Stresses. The Open Horticulture Journal, 3, 21-30. https://doi.org/10.2174/1874840601003010021

[45] Anantharaju, P. and Muthiah, A.R. (2008) Screening for Drought Tolerance in Cowpea Vigna unguiculata (L.) walp. Legume Research, 31, 283-285.

[46] Iwuagwu, M., Ogbonnaya, C.I. and Onyike, N.B. (2017) Physiological Response of Cowpea [Vigna unguiculata (L.) Walp.] to Drought: The Osmotic Adjustment Resistance Strategy Physiological Response of Cowpea [Vigna unguiculata (L.) Walp.]. Academic Journal of Science, 7, 329-344.

[47] Islam, S., Calvin, K. and Willy, V. (2011) Physiological and Morphological Characteristics of Cowpea Genotypes to Drought Stress. Arkansas Environmental, Agricultural and Consumer Sciences Journal, 42-45.

[48] Dadson, R.B., Hashem, F.M., Javaid, I., Joshi, J., Allen, A.L. and Devine, T.E. (2005) Crop/Stress Physiology Effect of Water Stress on the Yield of Cowpea (Vigna unguiculata L. Walp.) Genotypes in the Delmarva Region of the United States. Journal of Agronomy and Crop Science, 191, 210-217. 
https://doi.org/10.1111/j.1439-037X.2005.00155.X

[49] Agbicodo, E.M., Fatokun, C.A., Muranaka, S., Visser, R.G.F. and Linden Van Der, C.G. (2009) Breeding Drought Tolerant Cowpea: Constraints, Accomplishments, and Future Prospects. Euphytica, 167, 353-370.

https://doi.org/10.1007/s10681-009-9893-8

[50] Turk, K.J. and Hall, A.E. (1980) Drought Adaptation of Cowpea. II. Influence of Drought on Plant Water Status and Relations with Seed Yield. Agronomy Journal, 72, 421-427. https://doi.org/10.2134/agronj1980.00021962007200030005x

[51] Anuradha, Goyal, R.K. and Bishnoi, C. (2017) Assimilate Partitioning and Distribution in Fruit Crops: A Review. Journal of Pharmacognosy and Phytochemistry, 6, 479-484. 